\title{
The role of attention in conscious recollection
}

\author{
Felipe De Brigard * \\ Department of Psychology, Harvard University, Cambridge, MA, USA
}

\section{Edited by:}

Jeroen J. A. Van Boxtel, University of California Los Angeles, USA

\section{Reviewed by:}

Giorgio Marchetti, Mind,

Consciousness, and Language, Italy

Guido Hesselmann, Charité Campus

Mitte, Germany

\section{*Correspondence:}

Felipe De Brigard, Department of

Psychology, Harvard University,

William James Hall 884, 33 Kirkland

Street, Cambridge, MA 02138, USA.

e-mail: brigard@wjh.harvard.edu
Most research on the relationship between attention and consciousness has been limited to perception. However, perceptions are not the only kinds of mental contents of which we can be conscious. An important set of conscious states that has not received proper treatment within this discussion is that of memories. This paper reviews compelling evidence indicating that attention may be necessary, but probably not sufficient, for conscious recollection. However, it is argued that unlike the case of conscious perception, the kind of attention required during recollection is internal, as opposed to external, attention. As such, the surveyed empirical evidence is interpreted as suggesting that internal attention is necessary, but probably not sufficient, for conscious recollection. The paper begins by justifying the need for clear distinctions among different kinds of attention, and then emphasizes the difference between internal and external attention. Next, evidence from behavioral, neuropsychological, and neuroimaging studies suggesting that internal attention is required for the successful retrieval of memorial contents is reviewed. In turn, it is argued that internal attention during recollection is what makes us conscious of the contents of retrieved memories; further evidence in support of this claim is also provided. Finally, it is suggested that internal attention is probably not sufficient for conscious recollection. Open questions and possible avenues for future research are also mentioned.

Keywords: attention, consciousness, memory, recollection, retrieval, global neuronal workspace

\section{INTRODUCTION}

Although few would deny that consciousness and attention are intimately intertwined, their precise relationship remains unclear. Generally speaking, opinions about the nature of their relationship fall within one of three general views. The first view holds that attention is neither necessary nor sufficient for consciousness (Lamme, 2003; Koivisto et al., 2005). According to this perspective, even though attention and consciousness regularly occur in tandem, under specific circumstances they can be separated, suggesting that, in fact, consciousness and attention are dissociable processes (Koch and Tsuchiya, 2007). It follows from this view that it is possible to attend to something one is not conscious of, just as it is possible to be conscious of something while not attending to it. The second view, in contrast, holds that attention is necessary and sufficient for consciousness (Posner, 1994; Prinz, 2000, 2011). According to this perspective, the mechanisms of consciousness and attention are not entirely dissociable - although it remains an open question whether the precise relationship between such mechanisms is that of identity, causality, or constituency (Block, in preparation). A consequence of this view is that one cannot be conscious of something unless one attends to it, just as one cannot attend to something and fail to be conscious of it. Finally, there is an intermediate position according to which attention is necessary but not sufficient for consciousness (Moran and Desimone, 1985; Merikle and Joordens, 1997; Rensink et al., 1997; Dehaene et al., 2006; Dehaene and Changeux, 2011). It follows from this view that one cannot be conscious of something unless one attends to it, but attending to something is not enough to make one conscious of it, insofar as other processes are required. As such, this third view agrees with the first one in that it denies that attention is sufficient for consciousness, while at the same time agrees with the second view in suggesting that attention is necessary for consciousness.

Since most research on attention has been limited to perception - which in turn is usually confined to vision and, to a lesser degree, audition - it is unsurprising to find that most of the discussion on attention and consciousness has focused on conscious perception. However, perceptual contents are not the only kind of mental contents of which we are ordinarily conscious. An important set of conscious mental states which has not been sufficiently addressed within this discussion, is that of memories. When we remember, we usually experience something akin to the reinstatement of the content of a previous experience, which may or may not have been perceptual ${ }^{1}$. To be sure, memory enables us to recall past visual or auditory experiences, but it also brings to mind old nightmares and long-gone aspirations. Unlike perception, which allows us to be consciously aware of our present, memory allows us to be consciously aware of our past. As a result, it is natural to wonder whether or not attention plays a role during conscious

\footnotetext{
${ }^{1}$ Strictly speaking, when we remember we not only experience something akin to the reinstatement of the content of the original experience but also the sense of having experienced such a content in the past. In the philosophy of memory there is some debate as to whether this "double-consciousness" is to be understood as the belief that what content of the memory portrays occurred to us in the past (e.g., Locke and Russell) or rather as an emotion with no cognitive content (e.g., William James, see Locke, 1971). In psychology and cognitive neuroscience these issues are usually studied under the rubric "metamemory." Since the purpose of the present paper is to understand the role of attention in the conscious recollection of memorial contents, this interesting metamemory issue will be sidestepped.
} 
recollection, and also whether or not that role is analogous to the role it plays during conscious perception. Does conscious recollection depend in any way on attention or are they independent processes? More generally, what is the relationship between attention and consciousness during conscious recollection?

In this paper I want to defend the claim that attention is necessary, but probably not sufficient, for conscious recollection. However, unlike the case of conscious perception, I argue that the kind of attention required during recollection is internal, as opposed to external, attention. This makes the role of attention during conscious recollection significantly similar, but also importantly different, from the role it plays during conscious perception. More precisely, then, I argue that internal attention is necessary, but probably not sufficient, for conscious recollection. To that end, in Section "Varieties of Attention" I start by justifying the need for clear distinctions among different kinds of attention, emphasizing the difference between internal and external attention. In Section "Internal Attention and Episodic Memory Retrieval," I review evidence from behavioral, neuropsychological, and neuroimaging studies suggesting that internal attention is required for the successful retrieval of memorial contents. Next, in Section "Internal Attention is Necessary for Conscious Recollection," I argue that internal attention during recollection is what makes us conscious of the contents of retrieved memories. In turn, in Section "Internal Attention May Not be Sufficient for Conscious Recollection," I briefly argue for the probable non-sufficiency of internal attention for conscious recollection. I conclude with some questions for future research.

\section{VARIETIES OF ATTENTION}

When James remarked that "everyone knows what attention is" (James, 1890), he was rather overconfident. In reality, there seems to be a substantial amount of disagreement as to what the nature of attention is (Styles, 1997). Part of the problem is that neither the folk nor the scientific use of the term "attention" is sufficiently precise. We usually employ the term "attention" in non-scientific contexts to refer to a wide array of phenomena. The word "attention" sometimes refers to the way in which we engage in certain cognitive tasks; like when we play chess attentively rather than distractedly. Other times "attention" means bringing something to the foreground of the mind, as when we mentally single out the player with the ball when watching a soccer game. Yet, on other occasions, we use the term "attention" to explain why we were not aware of certain information - as when we justify our failing to remember someone's remark, or our inability to recognize a particular street when ambling absentmindedly, by simply saying that we were not paying attention. The problem is not with our use of the term "attention" in such circumstances. The trouble is rather that "attention" is not the only term we can employ to convey the same message. In certain contexts we use terms like "perceiving," "noticing," "being aware of," and even "being conscious of" when we could have easily used the term "attending" instead. Given people often use these terms interchangeably (De Brigard, 2010), this lack of semantic precision between the words "consciousness" and "attention" becomes more problematic when trying to identify the relationship between the two folk psychological notions, "consciousness," and "attention."
Semantic consensus is not found in scientific circles either. On the one hand, there is disagreement as to whether "attention" refers to a personal or a sub-personal phenomenon (Watzl, 2011). Specifically, there is disagreement as to whether attention refers to a process one should expect to find a neural correlate for; or whether it refers to something the person does in virtue of having a brain, but for which it would be a category-mistake to try to find a neural correlate (Mole, 2010; Wu, 2011). On the other hand, there is disagreement as to whether or not attention names a natural kind. For instance, some suggest that attention does not name a single cognitive mechanism, but rather denotes particular ways in which certain cognitive processes can be carried out. Listening attentively and observing attentively are not two different processes (i.e., audition and vision) that share a common third mechanism (i.e., attention); they are simply two different cognitive processes, carried out in distinctly precise ways that may or may not share common properties (Parasuraman, 2000; Duncan, 2006). As such, it would be a mistake to try to find the neural correlate of attention per se, independently of other cognitive processes. In contrast, one could see attention as a unified cognitive process with either an identifiable sub-personal neural correlate (Prinz, 2011), or a set of personal-level phenomena such as behaviors (Wu, 2011) or subjective mental contents (Smithies, 2011; Watzl, 2011). Those who consider attention reducible to a neural process face the daunting task of identifying a single brain mechanism responsible for all forms of attentive behavior. Likewise, those who think that attention could be identified with a series of personal-level phenomena face the difficult task of discerning necessary and sufficient conditions for behaviors or subjective states to qualify as instances of attention.

Employing different methods, many cognitive neuroscientists working on attention adopt a reductionist approach. Critics of this approach claim that extant empirical evidence strongly suggests that there may not be a single neural mechanism responsible for all forms of attention (Wu, 2011). The lack of a common neural denominator for all forms of attention would make it tempting to advocate either for anti-reductionism - so that we are to find the essence of attention at the personal rather than the sub-personal neuronal level (Mole, 2010; Watzl, 2011) - or for eliminativism, ridding scientific psychology of the term "attention" (Allport, 1993; Anderson, 2011). But there is another alternative. "Attention" may not name a single unified neural mechanism. Indeed, it may not name a natural kind at all. Yet it may be worth keeping around in our scientific practice. After all, not all scientifically useful psychological terms refer to natural kinds, let alone single neural mechanisms. Consider memory. Memory researchers have struggled for decades to come up with a single unified definition of "memory"; something general enough to encompass different kinds of memory (i.e., semantic, episodic, implicit, etc.), but specific enough to separate it from other forms of cognitive and non-cognitive phenomena (Tulving, 2002). Additionally, extant scientific evidence conclusively shows that different forms of memory are subserved by different neural mechanisms (Schacter et al., 2000; De Brigard et al., in press). Nonetheless, despite longstanding disagreements as to what its essence may be, and despite its multiple and disjoint neural implementations, "memory" is still a useful term in scientific psychology as well as neuroscience. 
I believe "attention" might be just like "memory." In a recent review, Chun et al. (2011) put forth precisely this proposal. Consistent with the anti-reductionist spirit regarding attention, Chun et al. agree that there are multiple attentional systems that appear to be correlated with different neural mechanisms. But the fact that there may not be a single neural property shared by all attentional systems does not deter them from suggesting that all forms of attention share three essential properties at the computational level. First, according to Chun et al. (2011), attention is essentially a filtering process with limited informational capacity. Since the amount of information we live in exceeds our capacity to effectively process it, attention evolved to filter out irrelevant information detrimental to the ongoing cognitive or behavioral task (Pashler et al., 2001). Second, attention is essentially selective. In filtering out information for subsequent processing, attention necessarily selects informational items that will be further processed from those that will not. Finally, attention modulates the ease of processing of the selected information. Attended information is processed more efficiently and more deeply than information that is not selected by attentional mechanisms. Thus, according to Chun et al. (2011), all forms of attention share these three computational characteristics (i.e., filtering, selectiveness, and modulation), which may or may not be implemented by the same neural mechanism. Indeed, they suggest that trying to understand different kinds of attention in terms of their neural mechanisms may not be the best way to proceed. Instead, they suggest a taxonomy based on the type of information attention operates over; what they call "the targets of attention."

According to Chun et al. (2011) proposed taxonomy, attention can be captured, first and foremost, either by targets in one's surrounding environment (external) or within one's own mind (internal). Thus, external versus internal attention constitute the first taxonomical division. External attention refers to the filtering, selection, and modulation of externally generated sensory information, whereas internal attention refers to the filtering, selection, and modulation of internally generated information - in the form of representations containing information not directly linked to objects in one's immediate surrounding environment. It is worth noting that the division between internal and external attention is similar to, but also importantly different from, categorizations that have been proposed in the past. For instance, attention has been separated into exogenous and endogenous attention (Egeth and Yantis, 1997). Endogenous attention refers to the voluntary selection and modulation of information elicited by top-down mechanisms of orientation and control, such as one's goals and intentions. Conversely, exogenous attention refers to the involuntary and bottom-up driven allocation of attention onto a target that is noted or otherwise cognitively highlighted for reasons outside of one's control. Endogenous and exogenous attention, however, do not map onto the internal/external classification. After all, external objects can be attended both endogenously - as when we voluntarily and in a controlled manner direct our attention to a desired external target - and exogenously, as when a particular external target captures our attention involuntarily and in a mandatory fashion. Another popular division is between covert and overt attention (Wright and Ward, 2008). Overt attention refers to a shift in attentional allocation accompanied by noticeable eye movements; whereas covert attention refers to a shift in attentional allocation with the eyes fixed on a certain target. However, as with the endogenous/exogenous dichotomy, the covert/overt distinction does not map squarely onto the internal/external categorization either. For one, as it has been shown experimentally, it is possible to divert attention from one target onto another without concomitant saccadic movements (Juan et al., 2004) which, incidentally, evidences the fact that attention can be spread over a region of space and not only toward individual objects. Likewise, evidence shows that attention allocated to internally generated information is often times accompanied by eye movements (Hunt and Kingstone, 2003). Finally, it is extremely improbable that eye movements could provide a useful wedge to divide internal and external attention to non-visual stimuli. Therefore, mechanism-based dissociations such as endogenous/exogenous and covert/overt do not map onto the target-based distinction between internal and external attention suggested by Chun et al. (2011).

By embracing Chun et al.'s (2011) informational target- rather than a mechanism-based taxonomy for attention, I am committing to the very real possibility that internal and external attention may not share the same neural operations. This consequence already appears to be validated by recent studies showing dissociations between brain regions engaged during internal-monitoring tasks and brain regions involved in external orienting and detection tasks (e.g., Esterman et al., 2009). Moreover, it is also expected that more fine-grained distinctions within these categories, such as feature- versus object-based attention within external attention, will map onto different neural mechanisms. Given this variability in the neural implementation of different kinds of attention, it is difficult to assess general claims like "attention is necessary for consciousness" or "attention is sufficient for consciousness." If either of these claims is supposed to capture something about the relationship between the mechanisms of attention and consciousness, they must be modified so as to specify the kind of attention to which they are supposed to apply. Since we are concerned here with conscious recollection, where the information of which we are aware is internally generated, the relevant kind of attention is internal. However, in order to understand the role of internal attention in conscious recollection, it is essential to first explore its role during episodic memory retrieval.

\section{INTERNAL ATTENTION AND EPISODIC MEMORY RETRIEVAL}

As mentioned above, external attention involves the filtering, selection, and modulation of sensory information (Chun et al., 2011). In addition, external attention can be allocated to one or to several sensory modalities, it can be focal or distributed spatially, and it can be transient or sustained. Each one of these forms of external attention activates distinct brain mechanism, some of which share certain features. For instance, visual attention enhances retinotopical activation in the visual cortex (Tootell et al., 1998), while auditory attention does so tonotopically in the auditory cortex (Woldforff et al., 1993). Thus, although the relevant cortical areas of enhanced activation differ across modalities, the specific processing elicited by attention appears to be similar. Likewise, external attention is known to recruit a fronto-parietal network of activation (Corbetta and Shulman, 2002). However, 
it has been shown that the timing of this recruitment differs depending on whether attention is goal-directed or stimulusdriven. Goal-directed or "top-down" attention recruits frontal regions of the fronto-parietal network first; stimulus-driven or "bottom-up" attention recruits parietal regions first (Buschman and Miller, 2007). Thus, while both top-down and bottom-up attention recruit similar brain regions, the order in which these regions are recruited differs.

Unsurprisingly, the mechanisms responsible for internal and external attention have much in common. Internal attention, defined as the filtering, selection, and modulation of internally generated information, operates over representations of items and events that need not be in the subject's immediate environment. Paradigmatically, internal attention operates over representations entertained during decision-making and working-memory tasks, but also - as I shall argue below - during retrieval of episodic information from long-term memory. Studies on task selection, in which competing options are filtered, chosen, and maintained, have shown specific capacity limitations expected from internal attentional processes. For instance, when choices are produced in rapid succession, the second response is delayed if presented less than half a second after the first choice - an effect known as "psychological refractory period" (Pashler, 1994). This bottleneck effect parallels well-known external attention effects, such as the attentional blink, in which a perceptual stimulus goes unnoticed if presented in close succession. Moreover, neuroimaging studies have shown common recruitment of regions engaged during attentional blink and the psychological refractory period (Wong, 2002; Marois and Ivanoff, 2005; Hesselmann et al., 2011; Marti et al., 2012), suggesting again the involvement of a common mechanism. Further neuroimaging studies on task selection have shown recruitment of several brain regions also associated with external attention, such as the prefrontal and anterior cingulated cortices (Botvinick et al., 2001). The overlap between internal and external attention mechanisms is even greater during workingmemory tasks. For example, the maintenance of representations in working-memory modulates modality congruent sensory cortices (Serences et al., 2009) much the same way external attention modulates activation of sensory cortices during perception. Likewise, working-memory tasks are disrupted by material-congruent distraction tasks, suggesting - once again - recruitment of common mechanisms.

Despite their similarities, internal, and external attention differ in important respects. In an illuminating study, Nobre et al. (2004) directly compared brain activity associated with attentional orientation during a perceptual and a working-memory task. Although, as expected, both tasks recruited a common network of brain regions, some important differences emerged. In particular, the right inferior parietal cortex, extending onto posterior angular gyrus, was preferentially involved in the orientation of external attention. On the other hand, bilateral intraparietal sulcus, as well as right ventral and bilateral dorsolateral prefrontal cortices, were preferentially recruited in orientation shifts during the internal attention task. These results were corroborated and expanded upon by Esterman et al. (2009). Using multivoxel pattern classification (MVPA) analysis they trained a classifier that successfully identified subpopulations of neurons within the superior parietal lobule preferentially associated with either internal or external attention-related activity. Finally, recent evidence showing differences between neural regions recruited during internal and external attention tasks comes from a study by Sestieri et al. (2010). In this study, participants engaged in top-down attentional search tasks looking for stimuli that were either retrieved or perceived. A direct comparison showed preferential activation in the angular gyrus, extending rostrally toward supramarginal gyrus and dorsally toward the intraparietal sulcus, precuneus, and posterior cingulate cortex for the memory search task. In contrast, the medial and ventral banks of the posterior intraparietal sulcus, as well as the superior parietal lobule, were preferentially associated with the perceptual search task. Taken together, the evidence just surveyed suggests that even though there is substantial overlap between internal and external attention, there are important differences as well (for a recent review, see Chun and Johnson, 2011).

Notwithstanding the substantial commonalities in the neural activations between internal and external attention, recent behavioral evidence is starting to suggest that internal rather than external attention may play a fundamental role during memory retrieval. Barring a few exceptions (e.g., Johnston et al., 1970; Trumbo and Milone, 1971), until the mid-1980s most memory researchers thought that, while attention was critical during memory encoding, it was not necessary for episodic memory retrieval. Neuropsychological evidence favored this claim, insofar as patients with attentional deficits due to parietal lesions showed no impairments during memory tasks (Critchley, 1953). Similar conclusions were reached by researchers conducting studies in which attention was manipulated during memory retrieval. In a classic paper, Baddeley et al. (1984) conducted a series of experiments using different attention-demanding secondary tasks during both encoding and retrieval. They found that, during encoding, all attention-demanding secondary tasks consistently impaired subsequent memory tests relative to conditions in which attention remained undisrupted. However, during retrieval, the same secondary tasks left memory performance unscathed. As a result, Baddeley et al. (1984) suggested that memory retrieval was a relatively automatic and mandatory operation that did not require the allocation of attentional resources. Since then, numerous studies have confirmed and clarified the essential role attention plays during episodic memory encoding (for a review, see Chun and Turk-Browne, 2007).

This dominant view has been recently challenged by a series of innovative studies showing that, under certain conditions, divided attention during episodic retrieval can actually affect memory performance. In a pioneer study, Fernandes and Moscovitch (2000) showed that when people engage in a materialcongruent secondary task in a divided attention paradigm during retrieval, performance significantly decreases relative to a baseline in which the memory test is the only task. In a related study, Hicks and Marsh (2000) showed that under deep encoding conditions divided attention at retrieval significantly reduces successful recollection. Indeed, they argue that previous studies failed to find effects of divided attention during retrieval precisely because they used shallow as opposed to deep encoding strategies. As a result, Hicks and Marsh (2000) hypothesize that, 
consistent with the dual-process theory of recognition memory ${ }^{2}$, successful memory retrieval requires attention only when it is recollection- rather than familiarity-based (Yonelinas, 2002). The necessity of attention for material-congruent and recollectionbased memory retrieval was nicely confirmed by Skinner and Fernandes (2008) who, employing a Remember/Know paradigm typically used to tap at differences between recollection and familiarity, showed that divided attention during retrieval only affected "Know" responses for material-congruent items. When the secondary task involved contents that differed from the target material (e.g., numerical tasks during retrieval of verbal information), and such materials were shallow versus deeply encoded, divided attention did not affect memory performance. Finally, Lozito and Mulligan (2006) extended these results by showing that, under conditions of strategic encoding (that need not be semantic) divided attention produces detrimental effects at retrieval. Taken together, the results of these - and related (e.g., Fernandes et al., 2005; Skinner et al., 2009) - studies suggest that divided attention affects recollection of strategic, deeply encoded information when attention is directed to material-congruent contents.

In addition to using shallow encoding strategies, previous studies failed to find an effect of attention during recollection for another reason: they either employed attention-diverting tasks with external targets (e.g., serial search) rather than internal targets, or they used material-incongruent tasks (e.g., number counting in verbal tasks) that did not demand the use of resources that internal attention was allocating to the process of recollecting memories. However, when the concurrent attentiondiverting task employed during memory retrieval targeted internal and material-congruent contents, recollection was significantly impaired. Consequently, focusing one's internal attention upon the to-be-retrieved material appears to be necessary for successful recollection of episodic memories. Indeed, this claim is further supported by recent neuropsychological studies on patients with parietal damage. As mentioned above, the traditional view is that patients with parietal lesions do not exhibit memory deficits. However, recent studies suggest that when recollection requires demanding internal maintenance and monitoring of retrieved information, patients with parietal damage show significant impairments relative to healthy controls. For instance, Berryhill et al. (2007) reported that, when compared with healthy controls, patients with bilateral ventral parietal lesions showed reduced levels of free-recall during autobiographical memory tasks as opposed to cued-recall, where they show no impairment. In addition, when compared with matched controls, patients

\footnotetext{
${ }^{2}$ According to the dual-process theory, recognition consists of two independent processes: recollection and familiarity. On the one hand, recollection involves the effortful retrieval of information about the encoded material plus contextual associations. Remembering the details of where you parked your car, its precise location and the visual layout of the surrounding environment constitute an example of a recollection-based memory. On the other hand, familiarity involves the mere feeling of having encountered the recognized item before, but without the capacity of conjuring up any details associated to such item. The feeling that you know someone you run into at a party without being able to place where you met her or what her name may be is an example of a familiarity-based memory (for a review, see Yonelinas, 2002).
}

showed decreased levels of vividness and number of details in their recollections during free- as opposed to cued-recall.

Curiously, when considered from the point of view of freerecall, a classic study conducted by Bisiach and Luzzatti (1978) appears to be consistent with the claim that parietal damage impairs voluntary retrieval of stored information. Bisiach and Luzzatti (1978) asked a patient with a parietal lesion resulting in severe hemispatial neglect, to remember the main square in Milan, the city in which he had lived all his life. Despite claiming to know the square quite well, the patient's report omitted the buildings to the left of the square when he tried to remember it facing one direction. When asked to imagine crossing the square and turning back, so he would be now facing the other direction from the opposite side, he omitted the buildings to his left even though he had just reported them. This surprising observation strongly suggests that damage to the parietal cortex, critical for the selection, and maintenance of visual information in external attention tasks, is also critical for the voluntary selection and maintenance of internal information during memory retrieval in conditions of free-recall.

The involvement of the parietal cortex during episodic memory retrieval has been a systematic finding in neuroimaging studies. For that reason, some theorists suggest that the role the parietal cortex may be playing during recollection is tantamount to the role it plays during visual perception. One of the most explicit articulations of this view has been put forth by Cabeza and colleagues (e.g., Cabeza, 2008; Cabeza et al., 2008). According to their "Attention to Memory" (AtoM) hypothesis, the dorsal parietal cortex, which is usually associated with top-down attention, is involved in voluntary, goal-directed attention, whereas the ventral parietal cortex, which is usually involved in bottom-up attention, appears to be associated with involuntary recollection (see Hutchinson et al., 2009, for some counter-evidence, but also Cabeza et al., 2011, for a response). Another hypothesis suggests that the parietal cortex may play a role analogous to the working-memory buffer suggested by Baddeley et al. (1998), insofar as it is required for gating stored information for decision-making and action (Wagner et al., 2005). Finally, one recent hypothesis - the Cortical Binding of Relational Activity (CoBRA) - suggests that the parietal cortex may modulate the reactivation of disaggregated sensory components during retrieval in order to bind them in the unified whole we experience during recollection (Shimamura, 2011). Although the jury is still out as to which of these views best captures the role of the parietal cortex during memory retrieval, for the present purposes it suffices to say that they all agree in that it plays a critical role in the selection (either voluntary or involuntary), modulation (either top-down or bottom-up), and maintenance of internally generated information - which, according to the operational definition used above, means that it plays a critical role during internal attention to memory representations.

In sum, the evidence reviewed in this section suggests that internal attention is required for the retrieval of episodic memories. Behavioral studies using divided attention paradigms show that when internal attention to material-congruent deeply encoded information is disrupted during retrieval, recollection is significantly impaired. In addition, neuropsychological studies in patients with parietal cortex damage, which usually results in attentional impairments to external stimuli, also suggest that 
under free-recall conditions they tend to retrieve less perceptual details from their autobiographical memories relative to both cued-recall and healthy controls. Finally, extant data coming from neuroimaging studies shows the involvement of attention-related parietal regions during episodic retrieval, further supporting the idea that internal attention plays a critical role during recollection. However, even if attention is required for episodic retrieval, there is still a further question as to whether it is necessary for conscious recollection - that is, the subjective experience of reliving the retrieved memory. In the next section I argue that this question should be answered in the affirmative.

\section{INTERNAL ATTENTION IS NECESSARY FOR CONSCIOUS RECOLLECTION}

The evidence reviewed so far suggests that internal attention is required for episodic memory retrieval. I now want to suggest that internal attention is also a necessary mechanism by means of which we become conscious of successfully retrieved memories. As mentioned before, episodic recollection requires the orchestrated operations of several brain regions. First, neuropsychological and neuroimaging studies show that the prefrontal cortex is involved in the initiation, monitoring, and maintenance of the retrieval attempt (Buckner and Wheeler, 2001). In particular, it has been suggested that the ventrolateral regions of the prefrontal cortex are involved in the initiation and maintenance of episodic memory retrieval, while the dorsolateral regions have been associated with the updating and manipulation of retrieved features (Wagner, 2002; Cabeza and St. Jacques, 2007). Second, recent evidence suggests that the medial-temporal lobes - previously thought to be involved only during memory encoding (but see Squire, 2004) - are also required for the successful binding and accessing of relational information from the neocortex during memory retrieval (Gilboa et al., 2005; Moscovitch et al., 2006). Finally, as mentioned in the previous section, it is now well accepted that the parietal cortex is involved in memory retrieval. Although its precise role remains elusive, extant theories suggests that it plays a role in the filtering and selection of information distributed in the sensory cortices (Shimamura, 2011).

It has also been suggested that this prefrontal/medialtemporal/parietal network of activation associated with episodic memory retrieval plays a critical role in the informational processing that gives rise to conscious awareness of mental contents. This suggestion has been thoroughly developed within the influential framework of the Global Neuronal Workspace (GNW) model suggested by Dehaene and Changeux (2000; see also Dehaene et al., 2003, 2006; Changeux and Dehaene, 2008; and Dehaene and Changeux, 2011). Briefly stated, the GNW model postulates two computational spaces in the brain, characterized by different patterns of connection. On the one hand, there is a processing network, which is seen as a set of local, informationally encapsulated, functionally specialized, and domain specific processors with limited numbers of medium-range connections. On the other hand, there is the GNW, which is characterized by distributed sets of cortical networks with long-range excitatory and inhibitory connections, allowing them to send and receive projections from distant areas in a global and flexible manner, so that the information these networks processes is neither encapsulated nor domain specific.
The projections that compose the GNW originate from pyramidal cells from layers II and III, the number of which is particularly high in lateral prefrontal, parietal, and medial-temporal cortices, specifically in the hippocampus, entorhinal, perirhinal, and parahippocampal cortices (von Economo, 1929; see also Changeux and Dehaene, 2008). Thus, according to the GNW hypothesis, informational inputs that enter the global neuronal workspace constitute the mental contents of which we are consciously aware.

The claim that attention is necessary for retrieved memories to become conscious becomes clear when it is considered from the point of view of the GNW hypothesis. Take the case of conscious recollection during free-recall. Assuming that what we know about the neural correlates of recollection is roughly accurate, the lateral prefrontal cortex would presumably initiate the process of retrieval (Rugg et al., 2002). Information is thus projected onto the ventral parietal cortex as well as the hippocampal and parahippocampal gyri, where stored indices of distributed sensory information would enable the binding of disaggregated memory traces (Nadel and Moscovitch, 2001). Then, dorsal regions of the parietal cortex would support the maintenance of the selected information via amplifying the signal from the local processing networks where it resides. When the signal reaches a certain threshold - most likely within the gamma frequency of 30-100 Hz (Jensen et al., 2007; see below) - the local sensory information that forms the memory trace would be broadcasted onto the global neuronal workspace which, by the GNW hypothesis, renders the memory not only conscious but also available for action. Since attention appears to operate via neural synchronization (e.g., Steinmetz et al., 2000), it follows that attention is the mechanism required to enhance gamma-band responses in local processing networks, which in turn renders them available for broadcasting onto the global neuronal workspace. Since these local processing networks represent stored rather than externally generated information from the immediate surrounding environment, the kind of attention required to render it available to the global neuronal workspace is internal instead of external attention. Thus, internal attention turns out to be necessary for conscious recollection.

Evidence in favor of the claim that internal attention permits the broadcasting of locally processed memory representations onto consciousness, comes from several electrophysiological and neuropsychological studies. As previously mentioned, attention appears to act upon local networks by modulating their synchronized firing (Steinmetz et al., 2000). Neuronal synchronization increases neuronal firing, which in turn promotes synaptic plasticity (Wespatat et al., 2004). Such neuronal changes have been correlated with increases in the gamma frequency of the relevant local network, which may explain why gamma-frequency activity predicts successful encoding during memory tasks, as confirmed by numerous EEG and MEG studies (e.g., Sederberg et al., 2003; Paller et al., 2009). Critically, increases in gamma activity have also been correlated with successful retrieval of old items versus correct rejection of new items (Gruber et al., 2004; Osipova et al., 2006; for a review see Jensen et al., 2007). Moreover, in a recent study involving intracranial electroencephalographic recordings in 52 patients with epilepsy, Sederberg et al. (2007) discovered that the same pattern of gamma-frequency activity that predicts successful encoding reappears at retrieval. Of note, this 
oscillatory activity emerges in the prefrontal cortex and the hippocampus, and then spreads onto the sensory cortex - an observation consistent with several of the aforementioned neuroimaging results on memory retrieval. This finding, coupled with previous results showing the involvement of parieto-occipital regions in the modulation of gamma-frequency activity during recollection (Osipova et al., 2006), gives further support to the claim that the prefrontal/medial-temporal/parietal cortex plays a critical role in gating information from local sensory networks onto the global neuronal workspace.

Behavioral studies conducted with individuals who suffered parietal lesions give further support to the claim that internal attention gates memories into consciousness. If, as hypothesized, parietal regions modulate the availability of local sensory representations onto the global neuronal workspace, one should expect a diminished sense of "re-experiencing" or "autonoetic consciousness" in patients whose parietal lesions hinder such broadcasting. Indeed, this prediction has been recently confirmed. Berryhill et al. (2007) tested autobiographical recollection in patients with bilateral parietal lesions and showed that, during free-recall, these individuals exhibited fewer episodic details and reported lower levels of vividness in their recollections, suggesting that a reduced number of sensory representations were actually made available to their conscious experience. In a related study, Davidson et al. (2008) reported that patients with parietal lesions showed a reduced number of "remember" responses, which are associated with increased subjective experience of recollection, relative to both "know" responses and controls. Drowos et al. (2010) also found reduced levels of "remember" relative to "know" responses in patients with parietal lesions using the Desee/Roediger-McDermott (DRM) paradigm. Finally, Simons et al. (2010) found that patients with bilateral parietal damage showed lower confidence levels for source recollection tasks, a result they interpret as suggesting that parietal lobe lesions impair subjective experience of episodic recollection. The view that internal attention is required for conscious recollection is entirely consistent with their interpretation.

Finally, the claim that internal attention is necessary for conscious recollection also finds support when one considers cuedrecall - albeit this foundation is perhaps more speculative. The fact that richer retrieval cues increase the likelihood of successful retrieval is at the heart of the notion of retrieval support, but it also suggests that these richer cues work precisely because they have a better chance of "highlighting" the relevant memory trace than poorer retrieval cues. This thought lies at the foundation of Tulving's (1982) classic synergistic ecphory model, according to which the subjective sense of recollection occurs when the memory trace interfaces with the retrieval cue - a process he, following Semon (1904), called "ecphory." Although little is known about the neural underpinnings of ecphory, research on memory reinstatement suggest that cued-recall facilitates the reactivation of regions engaged during encoding (Rugg et al., 1998, 2008). In a recent study, Manning et al. (2011) used electrocorticographic recordings in 69 patients during study and cued-recalled tests. They found that the recorded electrophysiological pattern of brain activity during encoding correlated with the pattern at retrieval. Critically, when successful reinstatement was evidenced it occurred within the gamma-band, suggesting the modulation of attentional mechanisms. This activity may be related to bottom-up attention, as suggested by Cabeza's (2008) AtoM model. It may also relate to the phenomenon of spontaneous autobiographical recollections that occur when unexpected stimulus, acting as powerful cues, manage to unintentionally trigger episodes from our past (Berntsen, 2010). Further research is needed to understand the precise ways in which bottom-up internal attention may render memories conscious. Nonetheless, the evidence reviewed in this section strongly suggests that internal attention is not only necessary to successfully retrieve episodic memories: it is also needed to render them conscious.

\section{INTERNAL ATTENTION MAY NOT BE SUFFICIENT FOR CONSCIOUS RECOLLECTION}

Although internal attention is necessary for retrieved contents to become conscious, recent evidence suggests that is not sufficient. There are two main reasons why this may be the case. The first reason is that successful episodic recollection requires that the memorial contents one internally attends to are effectively reinstated during retrieval. Striking evidence in favor of this claim comes from studies with patients suffering from visual amnesia. Rubin and Greenberg (1998) reported 11 cases of patients with focal lesions in occipital cortex. Although these patients did not exhibit retrograde or anterograde amnesia - as their medialtemporal lobes were preserved - they did show marked deficits in remembering visual details from their episodic autobiographical memories, the non-visual details of which they were still able to remember (Greenberg et al., 2005). Similar observations can be found in patients with certain kinds of visual agnosias, such as color and spatial location; these patients recollection of color and spatial details is impaired relative to their recollection of other preserved visual details, such as volume or directionality (Farah et al., 1988). If my rendition of the GNW model as it applies to conscious recollection is roughly correct, then we can find an explanation as to why these patients cannot access these particular informational contents consciously: it is not because they cannot attend to them, but rather because, when they try to, there is nothing to attend to. The damage in the occipital cortex makes it impossible to reinstate the sensory content which, had it been internally attended, would have been consciously recalled ${ }^{3}$.

Further support for this claim comes from a recent behavioral experiment conducted by Guerin et al. (2012). After studying a series of items, participants were presented with a recognition test in which they had to select one of three items. Critically, in one condition, participants saw two related items, both of the same kind as the studied item, plus a non-related item. None of the items was the studied item itself. In another condition, participants saw one non-related item and two related items, one of which was, in fact, the target item. Importantly, in the condition where the two

\footnotetext{
${ }^{3}$ In essence, this is the same explanation the GNW model offers to account for attentional blink (e.g., Dehaene et al., 2006). If the iconic memory of the masked stimulus in the occipital cortex has been erased and replaced by the masking item, when internal attention is reoriented toward the first content (i.e., the masked stimulus), it finds that such content is no longer available for conscious processing, so only the second content (i.e., the masking item) is broadcasted. I believe a similar phenomenon occurs during change blindness (for an explanation see De Brigard and Prinz, 2010).
} 
related items that did not include the target item, participants' false alarm rate was at baseline level; whereas, in the condition in which one of the two related items was the target item, participant's false alarm rate was significantly reduced. Eye-tracking data collected during this study showed that in both cases participants were selectively attending to the perceptual differences between the related items. However, given the difference in false alarms, it appears as though the use of attention to perceptually discriminate between two related foils was not sufficient for the accurate retrieval of the target item. However, once the content was reinstated - as when the target item was actually seen next to a foil - it was more likely to capture internal attention, rendering it accessible for conscious recollection. This result suggests that, in addition to directing one's internal attention to stored contents, the presence of such contents is required for attention to render them conscious during recollection. As a consequence, internal attention appears to be necessary but perhaps not sufficient for conscious recollection.

The second reason attention may not be sufficient for conscious recollection has to do with the fact that attention is not an all-or-nothing process. It may be possible that, being a modulatory mechanism, attention can render contents conscious only if a certain threshold is reached. Indeed, this is a fall-out of the GNW model (Dehaene et al., 2003). It has also been proven experimentally in numerous studies showing that, under specific conditions, certain stimuli can exhibit attentional-cuing effects even at the neuronal level - and yet those same stimuli go completely unnoticed by the subject (see, for instance, van Boxtel et al., 2010, for a review). The same may occur with memory traces that, for one reason or another, cannot reach the conscious threshold even when modulated by internal attentional mechanisms. In fact, it may be possible that unattended memory representations are responsible for certain priming effects as well as familiarity-based recognition judgments (Paller et al., 2009). Further research is needed to clarify the conditions under which internal attention to memory representations may suffice to render them conscious.

\section{CONCLUSION}

While this review only scratches the surface of a rather convoluted puzzle, I believe that the evidence surveyed in this paper strongly suggests that internal, as opposed to external, attention is necessary but maybe not sufficient for conscious recollection. There are still several open questions that deserve further scrutiny. Perhaps the most pressing one consists of defining the precise mechanisms involved in the kind of internal attention required for conscious recollection. Not only is there substantial disagreement as to the extent of the overlap between the neural correlates of external and internal attention (Chun and Johnson, 2011), there

\section{REFERENCES}

Allport, A. (1993). "Attention and control. Have we been asking the wrong questions?" in Attention and Performance XIV, eds D. E. Meyer and S. Kornblum (Cambridge: MIT Press), $182-218$.

Anderson, B. (2011). There is no such thing as attention.

is also disagreement as to the precise role each kind of attention plays during conscious experience. Another critical question concerns the role that attention plays during familiarity-based rather than recollection-based recognition (Yonelinas, 2002). As discussed, disturbing internal attention during retrieval affects recollection but not familiarity. Moreover, patients with parietal damage report significantly reduced numbers of "know" versus "remember" responses and lower confidence ratings, which are thought to track subjective feelings of remembering, suggesting that their recollective experience is impoverished (Davidson et al., 2008). Further research will be critical in illuminating the role that internal attention plays in differentiating recollection from familiarity.

Finally, it is also possible that the dispensability of internal attention during procedural memory performance could help us understand the difference between implicit and explicit memory (Schacter, 1992). Although much is known about the neural mechanisms responsible for these two kinds of memory, the precise role internal attention plays - if at all - during retrieval of implicit information is still understudied. In a pioneer study, Gooding et al. (1999) tested participants on an implicit word-stem completion test under divided attention conditions and found no differences in performance relative to full attention. Similar results were found using related paradigms, such as artificial-grammar learning tasks (Helman and Berry, 2003) and repetition priming (Clarke and Butler, 2008), supporting the hypothesis that attention does not play a critical role during the retrieval of implicit memory. Strong support in favor of this view comes from recent studies by Lozito and Mulligan (2010). Using a variety of implicit memory tasks - such as perceptual identification and category exemplar production tests - under divided attention conditions, Lozito and Mulligan (2010) found no effect of divided attention during implicit retrieval, and also no performance costs for the secondary task. To the best of my knowledge, the only study showing some reduction in priming during divided attention conditions at retrieval is Kinoshita (1999), who used a re-arranged word-stem completion task. As such, it remains a possibility that specific kinds of implicit tasks could require some level of attentional allocation. Further research is needed to understand this particular issue, and its relation to the more general question of the role of attention in conscious recollection.

\section{ACKNOWLEDGMENTS}

For helpful discussions while writing this paper I am grateful to Scott Guerin, Adrianne Harris, Nathan Spreng, and Daniel Schacter, whose "On the relation between memory and consciousness" (Schacter, 1989) planted the kernel from which some ideas expressed in this paper emerged.

memory. J. Exp. Psychol. Gen. 13, 518-540.

Berntsen, D. (2010). The unbidden past: involuntary autobiographical memories as a basic mode of remembering. Curr. Dir. Psychol. Sci. 19, 138-142.

Berryhill, M. E., Phuong, L., Picasso, L., Cabeza, R., and Olson, I. R.
(2007). Parietal lobe and episodic memory: bilateral damage causes impaired free recall of autobiographical memory. J. Neurosci. 27, 14415-14423.

Bisiach, E., and Luzzatti, C. (1978). Unilateral neglect of representational space. Cortex 14, 129-133. 
Botvinick, M., Carter, C. S., Braver, T. S., Barch, D. M., and Cohen, J. D. (2001). Conflict monitoring and cognitive control. Psychol. Rev. 108, 624-652.

Buckner, R. L., and Wheeler, M. E. (2001). The cognitive neuroscience of remembering. Nat. Rev. Neurosci. 2, 624-634.

Buschman, T. J., and Miller, E. K. (2007). Top-down versus bottom-up control of attention in the prefrontal and posterior parietal cortices. Science 281, 1185-1187.

Cabeza, R. (2008). Role of posterior parietal regions in episodic memory retrieval: the dual attentional process hypothesis. Neuropsychologia 46, 1813-1827.

Cabeza, R., Ciaramelli, E., Olson, I. R., and Moscovitch, M. (2008). The parietal cortex and episodic memory: an attentional account. Nat. Rev. Neurosci. 9, 613-625.

Cabeza, R., Mazuz, Y. S., Stokes, J., Kragel, J. E., Woldorff, M. G., Ciaramelli, E., Olson, I. R., and Moscovitch, M. (2011). Overlapping parietal activity in memory and perception: evidence for the attention to memory model. J. Cogn. Neurosci. 11, 3209-3217.

Cabeza, R., and St. Jacques, P. L. (2007). Functional neuroimaging of autobiographical memory. Trends Cogn. Sci. (Regul. Ed.) 11, 219-227.

Changeux, J., and Dehaene, S. (2008). "The neuronal workspace model: conscious processing and learning," in Learning and Memory: A Comprehensive Reference, ed. R. Menzel (London: Elsevier), 729-758.

Chun, M., Golomb, J., and TurkBrowne, N. B. (2011). A taxonomy of external and internal attention. Annu. Rev. Psychol. 62, 73-101.

Chun, M., and Johnson, M. K. (2011). Memory: enduring traces of perceptual and reflective attention. Neuron $72,520-535$.

Chun, M. M., and Turk-Browne, N. B. (2007). Interactions between attention and memory. Curr. Opin. Neurobiol. 17, 177-184.

Clarke, A. J. B., and Butler, L. T. (2008). Dissociating word stem completion and cued recall as a function of divided attention at retrieval. $\mathrm{Mem}$ ory $16,763-772$.

Corbetta, M., and Shulman, G. L. (2002). Control of goal-directed and stimulus driven attention in the brain. Nat. Rev. Neurosci. 3, 201-215.

Critchley, M. (1953). The Parietal Lobes. London: Edward Arnold.

Davidson, P. S. R., Anaki, D., Ciaramelli, E., Cohn, M., Kim, A., Murphy,
K. J., Troyer, A. K., Moscovitch, M., and Levine, B. (2008). Does lateral parietal cortex support episodic memory? Evidence from focal lesion patients. Neuropsychologia 46, 1743-1755.

De Brigard, F. (2010). Consciousness, attention and commonsense. J. Conscious. Stud. 17, 189-201.

De Brigard, F., Giovanello, K. S., and Kaufer, D. (in press). "Neuroanatomy of memory," in Behavioral Neurology and Neuropsychiatry, eds D. B. Arciniegas, C. A. Anderson, and C. M. Filley (Cambridge University Press).

De Brigard, F., and Prinz, J. J. (2010). Attention and consciousness. Wiley Interdiscip. Rev. Cogn. Sci. 1, 51-59.

Dehaene, S., and Changeux, J. (2000). Reward-dependent learning in neuronal networks for planning and decision making. Prog. Brain Res. 126, 217-229.

Dehaene, S., and Changeux, J. (2011). Experimental and theoretical approaches to conscious processing. Neuron 70, 200-227.

Dehaene, S., Changeux, J., Naccache, L. Sackur, J., and Sergent, C. (2006). Conscious, preconscious, and subliminal processing: a testable taxonomy. Trends Cogn. Sci. (Regul. Ed.) 10, 204-211.

Dehaene, S., Sergent, C., and Changeux, J. (2003). A neuronal network model linking subjective reports and objective physiological data during conscious perception. Proc. Natl. Acad. Sci. U.S.A. 100, 8520-8525.

Drowos, D. B., Berryhill, M. E., Andre, J., and Olson, I. R. (2010). True memory, false memory, and subjective memory after parietal lobe damage. Neuropsychology 24, 465-475.

Duncan, J. (2006). Brain mechanisms of attention. Q. J. Exp. Psychol. 59, 2-27.

Egeth, H. E., and Yantis, S. (1997). Visual attention: control, representation, and time course. Annu. Rev. Psychol. 48, 267-297.

Esterman, M., Chiu, Y., TamberRosenau, B. J., and Yantis, S. (2009). Decoding cognitive control in human parietal cortex. Proc. Natl. Acad. Sci. U.S.A. 106, 17974-17979.

Farah, M., Peronnet, F., Gonon, M. A., and Giard, M. H. (1988). Electrophysiological evidence for a shared representational medium for visual images and visual percepts. J. Exp. Psychol. Gen. 117, 248-257.

Fernandes, M. A., and Moscovitch, M. (2000). Divided attention and memory: evidence of substantial interference effects at retrieval and encoding. J. Exp. Psychol. Gen. 129 155-176.

Fernandes, M. A., Moscovitch, M., Ziegler, M., and Grady, C. (2005). Brain regions associated with successful and unsuccessful retrieval of verbal episodic memory as revealed by divided attention. Neuropsychologia 43, 1115-1127.

Gilboa, A., Ramirez, J., Kohler, S., Westmacott, R., Black, S. E., and Moscovitch, M. (2005). Retrieval of autobiographical memory in Alzheimer's disease: relation to volumes of medial temporal lobe and other structures. Hippocampus 15 535-550.

Gooding, P. A., Mayes, A. R., van Eijk, R., Meudell, P. R., and MacDonald, F. L. (1999). Do novel associate word stem completion and cued recall share the same memory retrieval processes? Memory 7 323-343.

Greenberg, D. L., Eacott, M. J., Brechin, D., and Rubin, D. C. (2005). Visual memory loss and autobiographical amnesia: a case study. Neuropsy chologia 43, 1493-1502.

Gruber, T., Tsivilis, D., Montaldi, D., and Müller, M. M. (2004) Induced gamma band responses: an early marker of memory encoding and retrieval. Neuroreport 15 1837-1841.

Guerin, S. A., Robbins, C. A., Gilmore, A. W., and Schacter, D. L. (2012) Retrieval failure contributes to gistbased false recognition. J. Mem Lang. 66, 68-78.

Helman, S., and Berry, D. C. (2003) Effects of divided attention and speeded responding on implicit and explicit retrieval of artificial grammar knowledge. Mem. Cognit. 31, 703-714.

Hesselmann, G., Flandin, G., and Dehaene, S. (2011). Probing the cortical network underlying the psychological refractory period: a combined EEG-fMRI study. Neuroimage 56, 1608-1621.

Hicks, J. L., and Marsh, R. L. (2000). Toward specifying the attentional demands of recognition memory. $J$. Exp. Psychol. Learn. Mem. Cogn. 26 1483-1498.

Hunt, A. R., and Kingstone, A. (2003) Inhibition of return: dissociating attentional and oculomotor components. J. Exp. Psychol. Hum. Percept. Perform. 29, 1068-1074.

Hutchinson, J. B., Uncapher, M. R., and Wagner, A. D. (2009). Posterior parietal cortex and episodic retrieval: convergent and divergent effects of attention and memory. Learn. Mem. $16,343-356$.
James, W. (1890). The Principles of Psychology. New York, NY: Henry Holt and Co.

Jensen, O., Kaiser, J., and Lachaux, J. (2007). Human gamma-frequency oscillations associated with attention and memory. Trends Cogn. Sci. (Regul. Ed.) 30, 317-324.

Johnston, W. A., Greenberg, S. N., Fisher, R. P., and Martin, D. W. (1970). Divided attention: a vehicle for monitoring memory processes. $J$. Exp. Psychol. Gen. 83, 164-171.

Juan, C. H., Shorter-Jacobi, S. M., and Schall, J. D. (2004). Dissociation of spatial attention and saccade preparation. Proc. Natl. Acad. Sci. U.S.A. 101, 15541-15544.

Kinoshita, S. (1999). Priming for novel associations: evidence for an attentional component. Memory 7 , 385-404.

Koch, C., and Tsuchiya, N. (2007). Attention and consciousness: two different processes. Trends Cogn. Sci. (Regul. Ed.) 11, 16-22.

Koivisto, M., Revonsuo, A., and Salminen, N. (2005). Independence of visual awareness from attention at early processing stages. Neuroreport $16,817-821$.

Lamme, V. A. F. (2003). Why visual attention and awareness are different. Trends Cogn. Sci. (Regul. Ed.) 7 , $12-18$.

Lozito, J. P., and Mulligan, N. W (2006). Exploring the role of attention during memory retrieval: effects of semantic encoding and divided attention. Mem. Cognit. 34, 986-998.

Lozito, J. P., and Mulligan, N. W. (2010). Exploring the role of attention during implicit memory retrieval. $J$. Mem. Lang. 63, 387-399.

Manning, J. R., Polyn, S. M., Baltuch, G. H., Litt, B., and Kahana, M. (2011). Oscillatory patterns in temporal lobe reveal context reinstatement during memory search. Proc. Natl. Acad. Sci. U.S.A. 108, 12893-12897.

Marois, R., and Ivanoff, J. (2005). Capacity limits of information processing in the brain. Trends Cogn. Sci. (Regul. Ed.) 9, 296-305.

Marti, S., Sigman, M., and Dehaene, S. (2012). A shared cortical bottleneck underlying attentional blink and psychological refractory period. Neuroimage 59, 2883-2898.

Merikle, P. M., and Joordens, S. (1997) Parallels between perception without attention and perception without awareness. Conscious. Cogn. 6, 219-236.

Mole, C. (2010). Attention is Cognitive Unison. Oxford: Oxford University Press. 
Moran, J., and Desimone, R. (1985). Selective attention gates visual processing in the extrastriate cortex. Science 229, 782-784.

Moscovitch, M., Nadel, L., Winocur, G., Gilboa, A., and Rosenbaum, R. S. (2006). The cognitive neuroscience of remote episodic, semantic and spatial memory. Curr. Opin. Neurobiol. 16, 179-190.

Nadel, M., and Moscovitch, M. (2001). The hippocampal complex and long-term memory revisited. Trends Cogn. Neurosci. (Regul. Ed.) 5, 228-230.

Nobre, A. C., Coull, J. T., Maquet, P., Frith, C. D., Vandenberghe, R., and Mesulam, M. M. (2004). Orienting attention to locations in perceptual versus mental representations. $J$. Cogn. Neurosci. 16, 363-373.

Osipova, D., Takashima, A., Oostenveld, R., Fernandez, G., Maris, E., and Jensen, O. (2006). Theta and gamma oscillations predict encoding and retrieval of declarative memory. $J$. Neurosci. 26, 7523-7531.

Paller, K. A., Voss, J. L., and Westerberg, C. E. (2009). Investigating the awareness of remembering. Perspect. Psychol. Sci. 4, 185-199.

Parasuraman, R. (2000). The Attentive Brain. Cambridge: MIT Press.

Pashler, H. (1994). Dual task interference in simple tasks: data and theory. Psychol. Bull. 116, 220-244.

Pashler, H., Johnston, J. C., and Ruthruff, E. (2001). Attention and performance. Annu. Rev. Psychol. 52, 629-651.

Posner, M. I. (1994). Attention: the mechanisms of consciousness. Proc. Natl. Acad. Sci. U.S.A. 91, 7398-7403.

Prinz, J. J. (2000). A neorofunctional theory of consciousness. Conscious. Cogn. 9, 243-259.

Prinz, J. J. (2011). "Is attention necessary and sufficient for consciousness?" in Attention: Philosophical and Psychological Essays, eds C. Mole, D. Smithies, and W. Wu (Oxford: Oxford University Press), 174-203.

Rensink, R. A., O’Regan, J. K., and Clark, J. J. (1997). To see or not to see: the need for attention to perceive changes in scenes. Psychol. Sci. 8, 368-373.

Rubin, D. C., and Greenberg, D. L. (1998). Visual memory-deficit amnesia: a distinct amnesic presentation and etiology. Proc. Natl. Acad. Sci. U.S.A. 95, 5413-5416.

Rugg, M. D., Fletcher, P. C., Allan, K., Frith, C. D, Frackowiak, R. S., and Dolan, R. J. (1998). Neural correlates of memory retrieval during recognition memory and cued recall. Neuroimage 8, 262-273.

Rugg, M. D., Johnson, J. D., Park, H., and Uncapher, M. R. (2008). Encoding-retrieval overlap in human episodic memory: a functional neuroimaging perspective. Prog. Brain Res. 169, 339-352.

Rugg, M. D., Otten, L. J., and Henson, R. N. (2002). The neural basis of episodic memory: evidence from functional neuroimaging. Philos. Trans. R. Soc. Lond. B Biol. Sci. 357, 1097-1110.

Schacter, D. L. (1989). "On the relation between memory and consciousness," in Varieties of Memory and Consciousness: Essays in Honor of Endel Tulving, eds H. L. Roediger III and F. I. M. Craik (Hillsdale, NJ: Erlbaum Associates), 355-389.

Schacter, D. L. (1992). Implicit knowledge: new perspectives on unconscious processes. Proc. Natl. Acad. Sci. U.S.A. 89, 11113-11117.

Schacter, D. L., Wagner, A. D., and Buckner, R. L. (2000). "Memory systems of 1999," in Handbook of Memory, eds E. Tulving and F. I. M. Craik (Oxford: Oxford University Press), 627-643.

Sederberg, P. B., Kahana, M. J., Howard, M. W., Donner, E. J., and Madsen, J. R. (2003). Theta and gamma oscillations during encoding predict subsequent recall. J. Neurosci. 23, 10809-10814.

Sederberg, P. B., Schulze-Bonhage, A., Madsen, J. R., Bromfield, E. B., Litt, B., Brandt, A., and Kahana, M. J. (2007). Gamma oscillations distinguish true from false memories. Psychol. Sci. 18, 927-932.

Semon, R. (1904). The Mneme. Leipzig: W. Engelmann.

Serences, J. T., Ester, E. F., Vogel, E. K., and Awh, E. (2009). Stimulusspecific delay activity in human primary visual cortex. Psychol. Sci. 20, 207-214.

Sestieri, C., Shulman, G. L., and Corbetta, M. (2010). Attention to memory and the environment: functional specialization and dynamic competition in human posterior parietal cortex. J. Neurosci. 30, 8445-8456.

Shimamura, A. P. (2011). Episodic retrieval and the cortical binding of relational activity. Cogn. Affect. Behav. Neurosci. 11, 277-291.

Simons, J. S., Peers, P. V., Mazus, Y. S., Berryhill, M. E., and Olson,
I. R. (2010). Dissociation between memory accurary and memory confidence following bilateral parietal lesions. Cereb. Cortex 20, 479-485.

Skinner, E., and Fernandes, M. A. (2008). Interfering with remembering and knowing: effects of divided attention at retrieval. Acta Psychol. (Amst.) 127, 211-222.

Skinner, E. I., Fernandes, M. A., and Grady, C. L. (2009). Memory networks supporting retrieval effort and retrieval success under conditions of full and divided attention. Exp. Psychol. 56, 386-396.

Smithies, D. (2011). "Attention is rational-access consciousness," in Attention: Philosophical and Psychological Essays, eds C. Mole, D. Smithies, and W. Wu (Oxford: Oxford University Press), 247-273.

Squire, L. R. (2004). Memory systems of the brain: a brief history and current perspective. Neurobiol. Learn. Mem. 82, 171-177.

Steinmetz, P. N., Roy, A., Fitzgerald, P. J., Hsiao, S. S., Johnson, K. O., and Niebur, E. (2000). Attention modulates synchronized neuronal firing in primate somato-sensory cortex. Nature 404, 187-190.

Styles, E. A. (1997). The Psychology of Attention. New York: Psychology Press.

Tootell, R. B. H., Hadjikhani, N., Hall, E. K., Marrett, S., Vanduffel, W., Vaughan, J. T., and Dale, A. M. (1998). The retinotopy of visual spatial attention. Neuron 21, 1409-1422.

Trumbo, D., and Milone, F. (1971). Primary task performance as a function of encoding, retention, and recall in a secondary task. J. Exp. Psychol. 91, 273-279.

Tulving, E. (1982). Synergistic ecphory in recall and recognition. Can. J. Psychol. 36, 130-147.

Tulving, E. (2002). Episodic memory: from mind to brain. Annu. Rev. Psychol. 53, 1-25.

van Boxtel, J. J. A., Tsuchiya, N. and Koch, C. (2010). Consciousness and attention: on sufficiency and necessity. Front. Psychol. 1:217. doi:10.3389/fpsyg.2010.00217

von Economo, C. (1929). The Cytoarchitectonics of the Human Cerebral Cortex. Oxford: Oxford University Press.

Wagner, A. D. (2002). "Cognitive control and episodic memory: contributions from prefrontal cortex," in Neuropsychology of Memory, eds L.
Squire and D. L. Schacter (New York: Guilford), 174-192.

Wagner, A. D., Shannon, B. J., Kahn, I., and Buckner, R. L. (2005). Parietal lobe contributions to episodic memory retrieval. Trends Cogn. Sci. (Regul. Ed.) 9, 445-453.

Watzl, S. (2011). The nature of attention. Philos. Compass 6, 842-853.

Wespatat, V., Tennigkeit, F., and Singer, W. (2004). Phase sensitivity of synaptic modifications in oscillating cells of rat visual cortex. J. Neurosci. 24, 9067-9075.

Woldforff, M., Gallen, C., Hampson, S. A., Hillyard, S., Pantev, C., Sobel, D., and Bloom, F. E. (1993). Modulation of early sensory processing in human auditory cortex during auditory selective attention. Proc. Natl. Acad. Sci. U.S.A. 90, 8722-8726.

Wong, K. F. E. (2002). The relationship between attentional blink and psychological refractory period. J. Exp. Psychol. Hum. Percept. Perform. 28, 54-71.

Wright, R. D., and Ward, L. M. (2008). Orienting of Attention. Oxford: Oxford University Press.

Wu, W. (2011). Confronting manymany problems: attention and agentive control. Nous 45, 50-76.

Yonelinas, A. (2002). The nature of recollection and familiarity: a review of 30 years of research. J. Mem. Lang. 46, 441-517.

Conflict of Interest Statement: The author declares that the research was conducted in the absence of any commercial or financial relationships that could be construed as a potential conflict of interest.

Received: 22 December 2011; accepted: 24 January 2012; published online: 10 February 2012.

Citation: De Brigard F (2012) The role of attention in conscious recollection. Front. Psychology 3:29. doi: 10.3389/fpsyg.2012.00029

This article was submitted to Frontiers in Consciousness Research, a specialty of Frontiers in Psychology.

Copyright () 2012 De Brigard. This is an open-access article distributed under the terms of the Creative Commons Attribution Non Commercial License, which permits non-commercial use, distribution, and reproduction in other forums, provided the original authors and source are credited. 